\title{
Orientational Analysis of the Vesic's Bearing Capacity of Shallow Foundations
}

\author{
L. F. dos Santos, A.C.de Freitas
}

\begin{abstract}
The determination of the bearing capacity of shallow foundations can be considered as a complex elasto-plastic deformation problem, which is often studied phenomenologically. The phenomenological equations are naturally constructed based on the typical dimensions of the variables involved (unit weight, foundation size, etc.) - all physical laws must guarantee the principle of dimensional balance. Nevertheless, there is another requirement physical laws must obey that is less often explored: all equations must be orientationally balanced. While this requirement is obvious for vectorial equations, the laws of continuum mechanics often mix tensors, vectors and scalar quantities. Moreover, not all scalar quantities are considered orientationless (for instance, areas and angles define an orientation determined by the unit vector normal to the plane). Here, the main equations found in the literature for the bearing capacity of soils will be analyzed, testing the orientational balance of the phenomenological equations. It will be shown that not all equations are well balanced - in particular the critical rigidity index for the generalized failure of soils determined by Vesic (1973) is not balanced. Perhaps not surprisingly, the equations that are well balanced lead to a good agreement with experimental data from the literature, while the critical rigidity index fails systematically when compared to tests in model foundations on sand.
\end{abstract}

Keywords: bearing capacity, critical rigidity index, orientational balance, shallow foundations.

\section{Introduction}

Analyzing the variables that govern a physical phenomenon, one may extract (at least partially) the mathematical relations between these variables. This is the principle behind the dimensional analysis, which provides the means to determine physical laws even before any analytical or phenomenological derivation is attempted. A complementary approach is provided by the orientational analysis.

This method is based upon assigning orientational symbols to physical quantities such as force, velocity and position, which are spatially oriented. While the orientational symbols are evident for these vector quantities, they may become rather intricate for tensorial quantities or even for some scalars, which may identify an orientation. These symbols sustain a multiplication rule that forms a noncyclic Abelian group with four members, and they can be used to derive additional information that resolves problems incompletely solved by conventional dimensional analysis.

The goal of this work is to provide the orientational analysis of the equations for the bearing capacity (Vesic, 1975), as well as the orientational analysis of the rigidity in$\operatorname{dex} I_{r}$ and the critical rigidity index $I_{r c r i t}$ which are proposed by Vesic for determining if a soil-foundation system will fail in a generalized or non-generalized mode.
The limit stress to generate soil rupture is called Terzaghi's equation (1943) for foundation shapes with $L>B$ (Vesic (1973), as shown in Fig. 1, has already proposed a shape correction factor for different foundation forms of $L>B$ ), given by:

$$
q_{\text {rup }}=c \cdot N_{c}+q \cdot N_{q}+\frac{1}{2} \gamma \cdot B \cdot N_{\gamma}
$$

where $c$ is the soil cohesion intercept, $q$ is the uniformly distributed load due to the overburden, $\gamma$ is the soil unit weight, $B$ is the smallest size of the foundation base and the $N_{c}, N_{q}$ and $N_{\gamma}$ are dimensionless bearing capacity factors, defined by:

$$
\begin{aligned}
& N_{c}=\left(N_{q}-1\right) \cot \phi \\
& N_{q}=e^{(\pi \tan \phi)}\left(\tan \left(\frac{\pi}{4}+\frac{\phi}{2}\right)\right)^{2} \\
& N_{\gamma}=2\left(N_{q}+1\right) \tan \phi
\end{aligned}
$$

These equations are stated for lower compressibility soils, i.e., idealized soil-foundation systems that fail as a rigid-plastic medium, instead of elasto-plastic. This idealization is considered by Vesic (1975) to be adequate as long as the rigidity index (Eq. 5) is larger than a critical rigidity index (Eq. 6).

Luiza Fernandes dos Santos, M.Sc. Researcher, Programa de Projeto de Estruturas, Escola Politécnica, Universidade Federal do Rio de Janeiro, Av. Athos da Silveira Ramos 149, Ilha do Fundão, Centro de Tecnologia - Bloco A, $2^{\circ}$ andar, Cidade Universitária, Rio de Janeiro, RJ, Brazil. e-mail: luizadosfs@gmail.com.

Alessandra Conde de Freitas, D.Sc., Associate Professor, Programa de Projeto de Estruturas, Escola Politécnica, Universidade Federal do Rio de Janeiro, Av. Athos da Silveira Ramos 149, Ilha do Fundão, Centro de Tecnologia - Bloco A, $2^{\circ}$ andar, Cidade Universitária, Rio de Janeiro, RJ, Brazil. e-mail: alessandracfreitas@ poli.ufrj.br. Submitted on October 1, 2017; Final Acceptance on February 13, 2020; Discussion open until August 31, 2020.

DOI: $10.28927 /$ SR.431003 


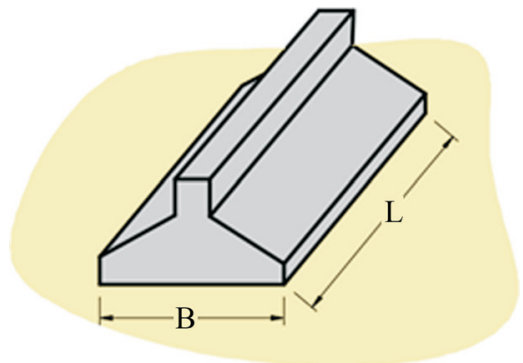

Figure 1 - Shallow foundation supported on a soil without overburden.

$$
\begin{aligned}
& I_{r}=\frac{G}{c+\sigma_{\text {med }} \tan \phi} \\
& I_{\text {r critical }}=\frac{1}{2} e^{\left(3.30-0.45 \frac{B}{L}\right) \cot \left(\frac{\pi}{4}-\frac{\phi}{2}\right)}
\end{aligned}
$$

where $G$ is the shear modulus, $\phi$ is soil friction angle and $\sigma_{\text {med }}$ is a characteristic stress scale, estimated as the mean stress at a depth $B / 2$ below the base of the footing. The shear modulus and mean stress can be defined respectively as:

$$
\begin{aligned}
& G=\frac{E}{2(1+v)} \\
& \sigma_{\text {med }}=\frac{\sigma_{v}^{\prime}+2 \sigma_{h}^{\prime}}{3}
\end{aligned}
$$

where $E$ is Youngs modulus, $v$ is the Poisson ratio and $\sigma_{v}$ and $\sigma_{h}$ are the effective vertical and horizontal stresses at a depth $B / 2$ below the base of the footing, respectively. The expressions for $\sigma_{v}$ and $\sigma_{h}$ are respectively:

$$
\begin{aligned}
& \sigma_{v}^{\prime}=\frac{1}{2} B \cdot \gamma \\
& \sigma_{h}^{\prime}=\sigma_{v}^{\prime} \cdot k_{0}
\end{aligned}
$$

where $k_{0}$ is the at-rest earth pressure coefficient and it is defined as

$$
k_{0}=1-\sin \phi
$$

The Eq. 11 applies only for sands.

\section{Material and Methods}

\subsection{Orientational analysis in general}

The symbol $\doteq$ will denote a orientational equality (Siano, 1985a), i.e., two quantities that carry the same orientational symbol will be related by the symbol $\fallingdotseq$. The symbols for orientations in the Cartesian directions $x, y$ and $z$ are respectively $l_{x}, l_{y}$ and $l_{z}$. Equivalent symbols and their multiplication table may be found for other coordinate systems (Siano, 1985b), but will not be necessary for our purpose here. Quantities without an orientation [some scalars (mass, time, ...) and tensor elements (normal stress, normal strain, ...)] will be denoted by the symbol $l_{0}$ (identity). To understand why quantities such as normal strain are deemed orientationless, the multiplication table for these symbols will be shown now.

The product between two quantities with different orientations will also have an orientation, following the respective multiplication table, which is analogous to the vector product rule, but commutative (without taking the signal into account), i.e.,

$$
\begin{aligned}
& l_{y} \cdot l_{z} \doteq \boldsymbol{l}_{\boldsymbol{x}} \doteq l_{z} \cdot l_{y} \\
& l_{x} \cdot l_{z} \doteq \boldsymbol{l}_{\boldsymbol{y}} \doteq l_{z} \cdot l_{x} \\
& l_{x} \cdot l_{y} \doteq \boldsymbol{l}_{z} \doteq l_{y} \cdot l_{x}
\end{aligned}
$$

There is also a rule of multiplication between two oriented quantities with same orientation, which always generates a quantity without orientation $\left(l_{0}\right)$ :

$$
\begin{aligned}
& l_{x} \cdot l_{x} \doteq l_{0} \\
& l_{y} \cdot l_{y} \doteq l_{0} \\
& l_{z} \cdot l_{z} \doteq l_{0} \\
& l_{0} \cdot l_{0} \doteq l_{0}
\end{aligned}
$$

The identity of this multiplication operation is $l_{0}$, such that multiplying it by any orientational symbol generates the same orientation:

$$
\begin{aligned}
& l_{x} \cdot l_{0} \doteq \boldsymbol{l}_{\boldsymbol{x}} \doteq l_{0} \cdot l_{x} \\
& l_{y} \cdot l_{0} \doteq \boldsymbol{l}_{\boldsymbol{y}} \doteq l_{0} \cdot l_{y} \\
& l_{z} \cdot l_{0} \doteq \boldsymbol{l}_{z} \doteq l_{0} \cdot l_{z}
\end{aligned}
$$

And finally, every symbol of orientation is the inverse of itself, that is,

$$
\begin{aligned}
& l_{0}^{-1} \doteq l_{0} \\
& l_{x}^{-1} \doteq l_{x} \\
& l_{y}^{-1} \doteq l_{y} \\
& l_{z}^{-1} \doteq l_{z}
\end{aligned}
$$
(Fig. 2)

It is possible to summarize all this in a single matrix

Therefore, these symbols and this multiplication operation form an Abelian group.

There is no definition of fractional powers of orientational symbols, so one may not have rational exponents in the original equation (physical law) when applying orientational analysis. In order to analyze the directionality of an equation which contains some root (fractional exponent), it is necessary to eliminate it by exponentiation.

For example, the orientational symbol of the area of the square $A$ is $l$, since

$$
A=L^{2} \doteq l_{x} \cdot l_{y} \doteq l_{z} \doteq A
$$




\begin{tabular}{c|cccc} 
& $1_{0}$ & $1_{x}$ & $l_{y}$ & $l_{z}$ \\
\hline$l_{0}$ & $l_{0}$ & $l_{x}$ & $l_{y}$ & $l_{z}$ \\
$l_{x}$ & $l_{x}$ & $1_{0}$ & $l_{z}$ & $l_{y}$ \\
$l_{y}$ & $l_{y}$ & $l_{z}$ & $l_{0}$ & $l_{x}$ \\
$l_{z}$ & $l_{z}$ & $l_{y}$ & $l_{x}$ & $l_{0}$
\end{tabular}

Figure 2 - General orientational matrix.

This also illustrates how a scalar quantity may bear an orientation symbol. In this example, the symbol is carried by the area of a square, which is a natural notion when dealing with the calculus of multivariate fields (for instance, the calculation of a flux in Gauss' theorem is dependent on the orientation of the area of the Gaussian surface of choice).

The fractional power would also be eliminated if the equation of the area represented as fractional power $(L=\sqrt{A})$ were raised to the fourth power:

$$
\begin{aligned}
& (L)^{4}=(\sqrt{A})^{4} \\
& L^{4}=A^{2}
\end{aligned}
$$

But this choice of exponent makes the orientational analysis less effective. Eqs. 15-18 show that even powers of any quantity will lead to the trivial conclusion $l_{0} \doteq l_{0}$. The form of Eq. 26 is referenced as the normal form, highlighting its usefulness for orientation analyses.

It can be concluded that the orientation of unknown quantities can be identified by balancing the orientational symbols derived from the physical law that defines this quantity.

\subsection{Orientational analysis of angles}

Angles are dimensionless, but they do carry an orientational symbol, so that equations associating vectors in different directions become directionally homogeneous, which is an important characteristic of orientational analysis.

An example of orientational angle analysis is found in the simple problem of the dynamics of a body in a tilted plane (Fig. 3).

Balancing the forces on the $x$ and $y$ axes leads to the conclusion that the friction coefficient is given by $\mu=\tan \theta$. The orientational analysis of the Amontons first law gives

$$
\begin{aligned}
& F_{a t}=\mu \cdot N \\
& l_{x} \doteq l_{\mu} \cdot l_{y}
\end{aligned}
$$

Therefore, by homogeneity,

$$
l_{\mu} \doteq l_{x} \cdot l_{y}^{-1} \doteq l_{z}
$$

with this, it can be concluded that $\tan \theta$ has direction $l_{z}$.

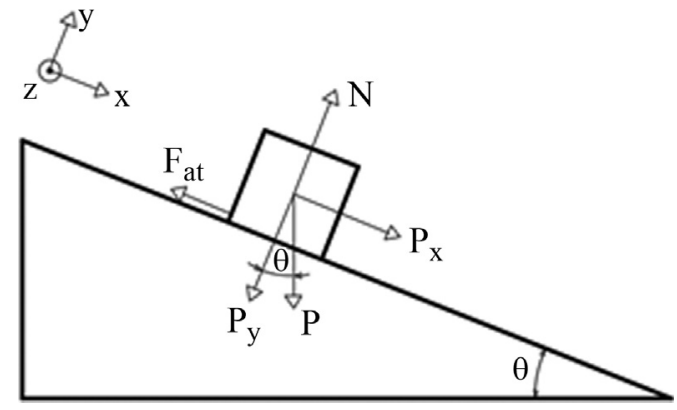

Figure 3 - Schematic of an inclined plane block.

\section{Results}

\subsection{Orientational analysis of bearing capacity factors for the Terzaghi's equation}

When analyzing Eq. 1, it can be verified that $N_{c}, N_{q}$ and $N_{\gamma}$ are adimensional:

$\left[q_{\text {rup }}\right]=[c] \cdot\left[N_{c}\right]+[q] \cdot\left[N_{q}\right]+\frac{1}{2}[\gamma] \cdot[B] \cdot\left[N_{\gamma}\right]$

$\left[\frac{M}{L T^{2}}\right]=\left[\frac{M}{L T^{2}}\right] \cdot\left[N_{c}\right]+\left[\frac{M}{L T^{2}}\right] \cdot\left[N_{q}\right]+\left[\frac{M}{L T^{2}}\right] \cdot\left[N_{\gamma}\right]$

Now, the orientational balance of the equations discussed in Siano (1985a) for $N_{c}, N_{q}$ and $N_{\gamma}$ (Eq. 2-4) will be analyzed. Since all three equations contain $\tan (\phi)$, and $N_{\gamma}$ contains $\left(\tan \left(\frac{\pi}{4}+\frac{\phi}{2}\right)\right)^{2}$, what will be discussed is how to obtain the orientational analysis of these functions. While the tangent function was already discussed, it is useful for other purposes to obtain its orientational symbol by analyzing its Taylor's series expansion. For both expressions, it can be written as:

$\tan \phi=\phi+\frac{\phi^{3}}{3}+\frac{2 \phi^{5}}{15}+\ldots$

$\left(\tan \left(\frac{\pi}{4}+\frac{\phi}{2}\right)\right)^{2}=1+2 \phi+2 \phi^{2}+\frac{5 \phi^{3}}{3}+\frac{4 \phi^{4}}{3}+\frac{61 \phi^{5}}{60}+\ldots$

If angles had a dimension ascribed to them, this expression would make no sense, since it would add distinct powers (for example, $\phi^{3}+\phi^{5}$ ). However, they can bear an orientational symbol. Indeed, since all powers in the Taylor expansion are odd, the orientation of the tangent function is the same as that of the friction angle $\phi$. Notice that Eq. 34 involves the square of a tangent function, which is orientationless. Indeed, its Taylor series expansion contains even and odd powers of $\phi$, and it cannot be balanced unless each term is orientationless (notice that the series terms containing odd powers of $\phi$ may still be orientationless, as long as the series coefficient has orientation).

Functions with no definite parity (which are neither even nor odd) need to have both dimensionless and directionless arguments. For example, $e^{x}$ 
$e^{x}=1+x+\frac{x^{2}}{2}+\frac{x^{3}}{6}+\frac{x^{4}}{24}+\ldots$

If $x \doteq l_{z}$, it can be concluded that the orientational balance of Eq. 35 is:

$$
\begin{aligned}
& l_{e^{x}} \doteq l_{0}+l_{z}+\frac{l_{z}^{2}}{2}+\frac{l_{z}^{3}}{6}+\ldots \\
& l_{e^{x}} \doteq l_{0}+l_{z}+l_{0}+l_{z}+\ldots
\end{aligned}
$$

which is not directionally balanced. Therefore,

$$
e^{x} \doteq l_{0} \text {, for } x \doteq l_{0}
$$

Frequently, an apparently orientational exponent is rendered orientationless by a pre-factor with a directionality that must be taken into account. This prefactor often contains $\pi$ (3.1415 ...), which is observed in the expression for $N_{q}$, since $e^{(\pi \cdot \tan \phi)}$ would violate the orientational balance, if $\pi$ had no direction. Because $\tan \phi \doteq l_{z}$, its can be concluded that $\pi \doteq l_{z}$ :

$$
\pi \tan \phi \doteq l_{z} \cdot l_{z} \doteq l_{0}
$$

and therefore

$$
e^{(\pi \tan \phi)} \doteq l_{0}
$$

The whole expression for $N_{q}$ is therefore balanced as

$$
N_{q}=e^{(\pi \tan \phi)}\left(\tan \left(\frac{\pi}{4}+\frac{\phi}{2}\right)\right)^{2}
$$

The balance of $N_{\gamma}$ is also obtained as

$$
\begin{aligned}
& N_{\gamma}=2\left(N_{q}+1\right) \tan (\phi) \\
& N_{\gamma} \doteq l_{0} \cdot\left(l_{0}+l_{0}\right) \cdot l_{z} \doteq l_{z}
\end{aligned}
$$

And finally, orientational analysis of $N_{c}$ gives

$$
\begin{aligned}
& N_{c}=\left(N_{q}-1\right) \cot (\phi) \\
& N_{c} \doteq\left(l_{0}-l_{0}\right) \cdot l_{z}^{-1} \doteq l_{0} \cdot l_{z} \doteq l_{z}
\end{aligned}
$$

The orientational balance of Eq. 1 will be checked by determining the orientational symbol for $q_{r u p}, q, c, \gamma$ and $B$. The coordinate system in Fig. 4 will be adopted, with the load applied vertically along the $y$ axis.

Some symbols are readily recognized as:

$$
\begin{aligned}
& \sigma=\frac{P}{A} \doteq \frac{l_{y}}{l_{x} \cdot l_{z}} \doteq \frac{l_{y}}{l_{y}} \doteq l_{0} \\
& q_{\text {rup }}=\frac{\text { weight of structure }}{\text { Area }} \doteq \frac{l_{y}}{l_{x} \cdot l_{z}} \doteq \frac{l_{y}}{l_{y}} \doteq l_{0}
\end{aligned}
$$
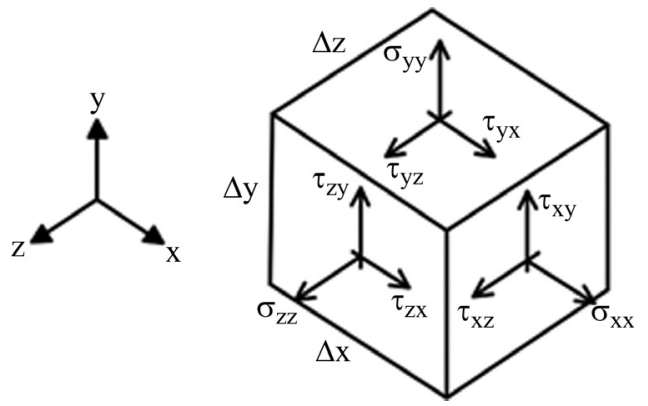

Figure 4 - Stress components in the Cartesian coordinate system.

$$
\begin{aligned}
& q=\frac{\text { weight of overburden }}{\text { Area }} \doteq \frac{l_{y}}{l_{x} \cdot l_{z}} \doteq \frac{l_{y}}{l_{y}} \doteq l_{0} \\
& \gamma=\frac{\text { weight of sand mas }}{\text { Volume }} \doteq \frac{l_{y}}{l_{x} \cdot l_{y} \cdot l_{z}} \doteq \frac{l_{y}}{l_{0}} \doteq l_{y} \\
& B \doteq l_{y} \\
& \sigma=\frac{P}{A} \doteq \frac{l_{y}}{l_{x} \cdot l_{z}} \doteq \frac{l_{y}}{l_{y}} \doteq l_{0}
\end{aligned}
$$

To determine the orientational symbol of $c$, its definition will be used in terms of the shear failure envelope curve, given by $\tau=c+\sigma \cdot \tan \phi$. This gives:

$$
\tau=c+\sigma \tan \phi \doteq c+l_{0} \cdot l_{z} \doteq c+l_{z}
$$

which is balanced only if $c$ has orientation $l_{z}$. Then, it is obtained by dimensional analysis of Eq. 1:

$$
\begin{aligned}
& q_{\text {rup }}=c \cdot N_{c}+q \cdot N_{q}+\frac{1}{2} \gamma \cdot B \cdot N_{\gamma} \\
& l_{0} \doteq l_{z} \cdot N_{c}+l_{0} \cdot N_{q}+l_{0} \cdot l_{y} \cdot l_{x} \cdot N_{\gamma} \\
& l_{0} \doteq l_{z} \cdot N_{c}+l_{0} \cdot N_{q}+l_{z} \cdot N_{\gamma}
\end{aligned}
$$

with this, it is concluded that the orientational symbol of $N_{q}$, $N_{\gamma}$ and $N_{c}$ should be respectively $l_{0}, l_{z}$ and $l_{z}$, in agreement with the results in Eqs. 41, 42 and 43. So Eq. 1 is directionally balanced.

\subsection{Orientational analysis of the Vesic equation for de- termination of generalized and non-generalized rup-} ture

As already shown in Eq. 5, the rigidity index, proposed by Vesic (1973), is given by:

$$
I_{r}=\frac{G}{c+\sigma_{m e d} \tan \phi}
$$

First, a orientational analysis of the Elasticity Theory equations must be performed, especially the relationship between stresses and strains. At first, normal strain does not carry a directionality 


$$
\varepsilon_{x}=\frac{\Delta L_{x}}{L_{x}} \doteq \frac{l_{x}}{l_{x}} \doteq l_{0}
$$

But $\varepsilon_{i j}$ is a tensor. In fact, for tensors, the diagonal elements have no direction, so that $\varepsilon_{x x} \doteq l_{0}$ is to be expected.

This helps to understand the direction of the elastic modules. Hooke's law can be expressed in three dimensions, where each normal strain is related to the three components of normal stress by the material properties, $E$ and $v$ :

$$
\begin{aligned}
& \varepsilon_{x}=\frac{1}{E}\left[\sigma_{x}-v\left(\sigma_{y}+\sigma_{z}\right)\right] \\
& \varepsilon_{y}=\frac{1}{E}\left[\sigma_{y}-v\left(\sigma_{x}+\sigma_{z}\right)\right] \\
& \varepsilon_{z}=\frac{1}{E}\left[\sigma_{z}-v\left(\sigma_{x}+\sigma_{y}\right)\right]
\end{aligned}
$$

Since the orientational symbols of $\sigma_{x}, \sigma_{y}, \sigma_{z}, \varepsilon_{x}, \varepsilon_{y}$ and $\varepsilon_{z}$, are $l_{0}$, as shown in Fig. 5 and Eq. 51 , then by balancing it can be seen that $E$ and $v$ are also $l_{0}$. With this, it can be concluded that $G$, shear modulus, also has direction $l_{0}$, since:

$$
\begin{aligned}
& G=\frac{E}{2(1+v)} \\
& G \doteq \frac{l_{0}}{2\left(1+l_{0}\right)} \doteq l_{0}
\end{aligned}
$$

Thus, the rigidity index (Eq. 5) has direction $l_{z}$, since:

$$
\begin{aligned}
& I_{r}=\frac{G}{c+\sigma_{\text {med }} \cdot \tan \phi} \\
& I_{r} \doteq \frac{l_{0}}{l_{z}+l_{0} \cdot l_{z}} \doteq \frac{l_{0}}{l_{z}+l_{z}} \doteq \frac{l_{0}}{l_{z}} \doteq l_{0} \cdot l_{z}^{-1} \doteq l_{z}
\end{aligned}
$$

In order to be comparable to $I_{r}$, the critical rigidity index of soil $I_{r \text { crit }}$ should be orientationally consistent, i.e., it should carry an orientational symbol $l_{z}$. However, when analyzing the orientational balance of this equation, a orientational inconsistency is found. According to Eq. 6, the critical rigidity index, for the cases of drained sand analysis, is given by:

$$
I_{\text {r critical }}=\frac{1}{2} e^{\left(3.30-0.45 \frac{B}{L}\right) \cot \left(\frac{\pi}{4}-\frac{\phi}{2}\right)}
$$

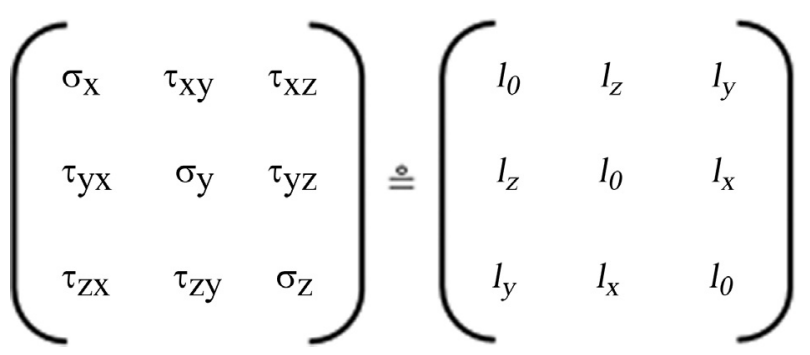

Figure 5 - Strain matrix and orientational deformation matrix.
Keeping the axes as in Fig. 4, the directions of the length and width of the foundation base are respectively $l_{B} \doteq l_{x}$ and $l_{L} \doteq l_{z}$.

As demonstrated previously in Eq. $38, e^{x} \doteq l_{0}$, for $x \doteq l_{0}$, and the exponent cannot be directionally non-trivial. Therefore $\left(3.30-0.45 \frac{B}{L}\right) \cot \left(\frac{\pi}{4}-\frac{\phi}{2}\right)$ should be $l_{0}$. But what happens is:

$$
\begin{aligned}
& \left(3.30-0.45 \frac{B}{L}\right) \cot \left(\frac{\pi}{4}-\frac{\phi}{2}\right) \doteq\left(l_{0}-l_{0} \cdot \frac{l_{x}}{l_{z}}\right) \cdot l_{\cot \left(\frac{\pi}{4}-\frac{\phi}{2}\right)} \\
& \left(3.30-0.45 \frac{B}{L}\right) \cot \left(\frac{\pi}{4}-\frac{\phi}{2}\right) \doteq\left(l_{0}-l_{0} \cdot l_{y}\right) \cdot l_{\cot \left(\frac{\pi}{4}-\frac{\phi}{2}\right)} \\
& \left(3.30-0.45 \frac{B}{L}\right) \cot \left(\frac{\pi}{4}-\frac{\phi}{2}\right) \doteq\left(l_{0}-l_{y}\right) \cdot l_{\cot \left(\frac{\pi}{4}-\frac{\phi}{2}\right)}
\end{aligned}
$$

The operation $\left(3.30-0.45 \frac{B}{L}\right)$ coton the right-hand side of Eq. 57 is inconsistent with orientational balancing, and therefore there must be something unphysical about this empirical critical rigidity index.

\section{Conclusions}

One may conclude that bearing capacity factors are directionally consistent. Often, a new proposal for a more accurate estimation of the bearing capacity factors $N_{c}, N_{q}$ and $N_{\gamma}$ is presented in the literature (Michalowski, 1997). The analysis presented here may provide a fast consistency check for these expressions. For instance, $N_{c}$ and $N_{\gamma}$ must be odd functions of the friction angle $\phi$, while $N_{q}$ must be even.

Although a orientational balance does not guarantee the accuracy of the equation, something may be stated about an unbalanced equation. For instance, the rigidity index equation for determining the limit between a generalized and a non-generalized rupture is not balanced. Vesic, in his article entitled "Bearing Capacity of Deep Foundations in Sand" (Vesic, 1963), shows experimental results in tests with circular and rectangular plates on the surface of the sandy soil. In each test, the rupture is classified as generalized, local or punching.

In Table 1, the experimental results are compared with the predictions from the analytical equation of critical rigidity index. The results shown in bold do not match those obtained experimentally. With this, it can be concluded that the criterion of Vesic is inaccurate in this regime. Furthermore, the injudicious use of this criterion jeopardizes the safety of the foundations, since a prediction of generalized failure when the soil is actually well-compressible may result in an overestimated ultimate load capacity (disregarding the necessary correction factor). The inadequacy of this equation could be anticipated by the orientational analysis of the proposed equation, which clearly raises doubts about this expression. 
Table 1 - Experimental results (Vesic, 1963) and the analytical result (Vesic, 1975).

\begin{tabular}{|c|c|c|c|c|c|c|c|c|}
\hline \multirow[t]{2}{*}{ Test \# } & \multicolumn{4}{|c|}{ Experimental results [Vesic (1963)] } & \multicolumn{4}{|c|}{ Rigidity Index criterion [Vesic (1975)] } \\
\hline & $B(\mathrm{~m})$ & $\gamma_{d}\left(\mathrm{kN} / \mathrm{m}^{3}\right)$ & $\phi\left(^{\circ}\right)$ & Failure & $E\left(\mathrm{kN} / \mathrm{m}^{2}\right)$ & $\left(I_{r}\right)_{c r i t}$ & $I_{r}$ & Failure \\
\hline 34 & 0.05 & 15.37 & 43.15 & General & $1,821.75$ & 359.58 & $3,463.46$ & General \\
\hline 21 & & 14.90 & 41.09 & Local & $1,820.36$ & 263.39 & $3,681.14$ & General \\
\hline 22 & & 14.38 & 38.85 & Local & $1,820.43$ & 192.85 & $3,934.37$ & General \\
\hline 23 & & 13.23 & 34.10 & Punching & $1,811.57$ & 107.58 & $4,581.91$ & General \\
\hline 44 & 0.10 & 15.50 & 43.72 & General & $2,476.09$ & 393.74 & $2,506.39$ & General \\
\hline 41 & & 15.02 & 41.61 & Local & $2,476.26$ & 284.25 & $2,665.09$ & General \\
\hline 42 & & 14.58 & 39.70 & Local & $2,479.70$ & 216.41 & $2,815.84$ & General \\
\hline 43 & & 13.34 & 34.54 & Punching & $2,464.05$ & 113.11 & $3,317.25$ & General \\
\hline 61 & 0.15 & 15.41 & 43.33 & General & $3,057.14$ & 369.95 & $2,053.11$ & General \\
\hline 62 & & 14.89 & 41.04 & Local & $3,059.30$ & 261.49 & $2,193.6$ & General \\
\hline 63 & & 14.69 & 40.18 & Local & $3,059.14$ & 231.33 & $2,250.15$ & General \\
\hline 64 & & 15.22 & 42.49 & Punching & $3,058.21$ & 324.55 & $2,103.16$ & General \\
\hline 84 & 0.20 & 15.41 & 43.33 & General & $3,530.08$ & 369.95 & $1,778.05$ & General \\
\hline 81 & & 15.25 & 42.62 & General & $3,531.21$ & 331.10 & $1,814.55$ & General \\
\hline 82 & & 15.25 & 42.62 & General & $3,531.21$ & 331.10 & $1,814.55$ & General \\
\hline 83 & & 14.09 & 37.63 & Local & $3,528.89$ & 164.46 & $2,105.97$ & General \\
\hline 16 & rectangular & 15.44 & 43.46 & General & $1,764.97$ & 903.27 & $3,542.85$ & General \\
\hline 1 & $0.051 \times 0.30$ & 14.99 & 41.48 & Local & $1,766.13$ & 640.82 & $3,750.94$ & General \\
\hline 2 & & 14.72 & 40.31 & Local & $1,766.21$ & 529.55 & $3,882.37$ & General \\
\hline 3 & & 13.45 & 34.98 & Punching & $1,759.65$ & 244.54 & $4,586.13$ & General \\
\hline
\end{tabular}

In Civil Engineering problems, the power of orientational analysis, unlike dimensional analysis, is not yet well explored and can lead to important advances. The mathematical structure of the laws of elasticity and plasticity allows their broad application and can guide efforts in determining appropriate empirical equations for quantities of interest.

The elastic modulus $E$ was not provided in Vesic (1963), but an expression is reported in Vesic (1973) associating this modulus to the mean normal stress $E=E_{1} \sqrt{\sigma_{m} / \sigma_{1}}$, with $E_{1}=39,180.65 \mathrm{kN} / \mathrm{m}^{2}\left(364 \mathrm{ton} / \mathrm{ft}^{2}\right)$ being the modulus at mean normal stress of $\sigma_{1}=$ $104.64 \mathrm{kN} / \mathrm{m}^{2}\left(1 \mathrm{ton} / \mathrm{ft}^{2}\right)$.

\section{References}

Michalowski, R.L. (1997). An estimate of the influence of soil weight on bearing capacity using limit analysis. Soils and Foundations, 37(4):57-64.

Siano, D.B. (1985a). Orientational Analysis - A Supplement to Dimensional Analysis - I. Journal of the Franklin Institute, 320(6):267-283.

Siano, D.B. (1985b). Orientational Analysis, Tensor Analysis and the Group Properties of the SI Supplementary
Units - II. Journal of the Franklin Institute, 320(6):285-302.

Terzaghi, K. (1943). Theoretical Soil Mechanics. John Willey \& Sons, New York.

Vesic, A.B. (1963). Bearing capacity of deep foundations in sand. Highway Research Record, 39:112-153.

Vesic, A.B. (1973). Analysis of ultimate loads of shallow foundations. J. Soil Mech. Found. Div., 99(1):45-73.

Vesic, A.B. (1975). Bearing Capacity of Shallow Foundations. Foundation Engineering Handbook, McGrawHill, New York, pp. 121-147.

\section{List of Symbols}

$B$ - smaller length of shallow foundations

$c$ - soil cohesion intercept

$E$ - Young's modulus

$G$ - shear modulus

$I_{r}$ - rigidity index

$I_{r \text { critical }}$ - critical rigidity index

$\mathrm{k}_{0}$ - coefficient of earth pressure at rest

$L$ - longer length of shallow foundations

$N_{c}, N_{q}, N_{\gamma}$ - bearing capacity factors

$q$ - uniformly distributed load

$q_{r u p}$ - rupture stress/ ultimate pressure

$\varepsilon$ - normal strain 
$\phi$ - friction angle
$\gamma$ - unit weight
$\gamma_{d}$ - unit weight of the dry soil

\author{
$v$ - Poisson ratio \\ $\sigma_{h}$ - horizontal stress \\ $\sigma_{\text {med }}$ - mean stress at a depth $B / 2$ below the base of the footing \\ $\sigma_{v}-$ vertical stress
}

